\title{
Displacement transmissibility characteristics of harmonically base excited damper isolators with mixed viscous damping
}

\author{
Xiaojuan Sun and Jianrun Zhang* \\ School of Mechanical Engineering, Southeast University, Nanjing, Jiangsu, China
}

Received 13 April 2012

Revised 22 August 2012

Accepted 1 April 2013

\begin{abstract}
The viscous damping force in the mixed form as $f_{d}(\dot{x})=c_{1} \dot{x}+c_{2}|\dot{x}| \dot{x}$ can well describe damping characteristics of isolators and dampers in many cases. In this paper, performance characteristics of single-degree-of-freedom (SDOF) linearstiffness isolators with mixed and piecewise mixed viscous damping are analytically examined under harmonic base excitation. Based on the first-order harmonic balance method (HBM), both relative and absolute displacement transmissibility expressions with the equivalent linear damping coefficient (ELDC) are given. And the analytical calculations show good agreement with the numerical results. Also, the influence of nonlinear damping on the response characteristics is investigated by comparing the transmissibility of linear and nonlinear systems. The resonant frequency always shifts to a lower value as the nonlinear damping component of the force $f_{d}(\dot{x})=c_{1} \dot{x}+c_{2}|\dot{x}| \dot{x}$ becomes stronger, and when the damping ratio in the corresponding linear model is relatively high, the relative transmissibility decreases at frequencies higher than the resonance frequency of the corresponding linear damping system and the absolute one increases for the frequency ratios above $\sqrt{2}$. Finally, the displacement transmissibility of a nonlinear isolator with piecewise mixed viscous damping is discussed and the process shows research similarity with the non-piecewise case.
\end{abstract}

Keywords: Mixed viscous damping, vibration isolation, harmonic balance method, equivalent linear damping coefficient, displacement transmissibility

\section{Introduction}

In order to improve the safety and comfort of the production, dampers or isolators with liquid are often used to control unwanted vibrations and noises in engineering [1-10]. A very basic description of a hydraulic damper is that it essentially consists of a piston moving in a closed cylinder, which has a small orifice in the piston head connecting either side through which the fluid flows $[3,9]$. Sometimes the damping force produced by liquid reciprocating across the damping orifice may be modeled by a combination of linear and quadratic damping terms relative to the mean fluid velocity as $f_{d}(\dot{x})=c_{1} \dot{x}+c_{2}|\dot{x}| \dot{x}$ instead of the proportional to the velocity for a laminar flow or to the square of the velocity for a turbulent flow [3,5,8]. Also, the damping force of a hydraulic engine/cab mount only with the inertia track or the orifice could be expressed as the above mixed viscous damping form when the properties of the main rubber were treated using the Kelvin-Voigt model and the liquid damping force in the track or the orifice was expressed as the quadratic term $f(\dot{x})=c|\dot{x}| \dot{x}[6,10]$. Besides, the mixed damping form was also used to model the

${ }^{*}$ Corresponding author: Jianrun Zhang, School of Mechanical Engineering, Southeast University, Nanjing 211189, Jiangsu, China. Tel.: +86 2552090501 8425; E-mail: zhangjr@seu.edu.cn. 
damping capacity of a metal-bonded rubber isolator [11]. Moreover, dampers were feasibly designed in piecewise damping characteristics [2,3,12].

For the treatment of the nonlinear damping force involving the quadratic term, there are various approaches. Guo et al. [1] evaluated the absolute displacement transmissibility of a single-degree-of-freedom (SDOF) base isolation system with a nonlinear viscous damping device using the Ritz-Galerkin method. Popov and Sankar [8] analysed the non-linear orifice type damper using the numerical integration. Nayfeh and Mook [13] solved the equation of motion for the unforced oscillator with quadratic damping using the averaging method which was generally suitable for weakly nonlinear problems. In practical engineering, a nonlinear damping device is often represented in the mathematical model by a linear viscous damper which provides a required equivalent viscous damping in the predesign stage. Commonly, the equivalent viscous damping coefficient, which is from an approximate solution based on the equal energy dissipation per cycles for linear viscous-damped and nonlinear-damped systems by assuming harmonic responses, is proposed [4,14]. Mallik [15] used the common method to treat nonlinear damping forces in the studies of a duffing oscillator with different damping mechanisms. Ravindra and Mallik [16] solved the equations with nonlinear restoring and damping forces by using the first-order harmonic balance method (HMB) which was applicable to both weakly and strongly nonlinear problems and the emphasis was placed on the effects of four typical damping models on the performance of isolation systems with different nonlinear restoring forces.

Displacement transmissibility is widely used in the design of vibration isolators to indicate the vibration transmission at different frequencies. For a base isolation system, both absolute displacement transmissibility and relative displacement transmissibility are often used as performance evaluations of the isolator [14]. To minimize the transmitted vibration from a vibrating foundation, the absolute displacement transmissibility is often examined to study the vibration isolation performance of nonlinear dampers or isolators $[1,8,16]$. Also, relative transmissibility is significant in an isolator under base excitation. For example, the relative displacement transmissibility is adopted as one of the suspension performance indices for the design of vehicle suspension systems $[17,18]$. The relative deflection is a measure of the clearance (known as working space, travel space or rattle space) required in the isolator, which should be bounded in the mechanical design. Milovanovic et al. [19] studied the performance of two SDOF isolators with cubic nonlinearities respectively in stiffness and damping terms under base excitation from the viewpoint of both absolute and relative displacement transmissibility.

This paper presents an analytical study on the harmonic responses of nonlinear damper isolators with mixed and piecewise mixed viscous damping and investigates the influence of the nonlinear damping on the displacement transmissibility. The absolute and relative displacement transmissibility expressions and the equivalent linear damping coefficient (ELDC) for a SDOF linear-stiffness isolation system with mixed viscous damping force as $f_{d}(\dot{x})=c_{1} \dot{x}+c_{2}|\dot{x}| \dot{x}$ are obtained based on the first-order HBM in Section 2 . The transmissibility expressions in terms of the ELDC, which reveals that the equivalent viscous damping coefficient has the first harmonic precision, have the forms of the linear isolation system. Compared with the results of the Runge-Kutta method in Section 3, the approximate solutions have high accuracy. Meanwhile, the effects of the nonlinear damping component on the two types of transmissibility are analytically studied by comparing nonlinear damping systems with different values of damping coefficients in the same peak transmissibility corresponding to a linear isolation system in Section 4. The comparisons show that the resonance frequency moves towards a smaller value in contrast with the linear damping model. And in the case of the corresponding linear system with relatively high damping ratio, the relative transmissibility of the nonlinear damping models changes lower at frequencies above the resonance frequency of the linear system and the absolute transmissibility changes higher for frequency ratios above $\sqrt{2}$. The stronger the nonlinear damping is, the more visible the above phenomena are. In Section 5, the similar analytical process is carried out to study a base isolation system with piecewise mixed viscous damping according to the sign of the relative velocity and it is presented that the two parameters $\xi_{11}+\xi_{21}$ and $\xi_{12}+\xi_{22}$ characterize both the ELDC and the displacement transmissibility.

\section{Approximate solutions of the SDOF isolation system with the mixed viscous damping}

Considering the SDOF vibration isolation system with mixed viscous damping subject to a base excitation shown in Fig. 1, the equation of motion for the mass $M$ is

$$
M \ddot{x}_{1}+k\left(x_{1}-x_{0}\right)+c_{1}\left(\dot{x}_{1}-\dot{x}_{0}\right)+c_{2}\left|\left(\dot{x}_{1}-\dot{x}_{0}\right)\right|\left(\dot{x}_{1}-\dot{x}_{0}\right)=0
$$




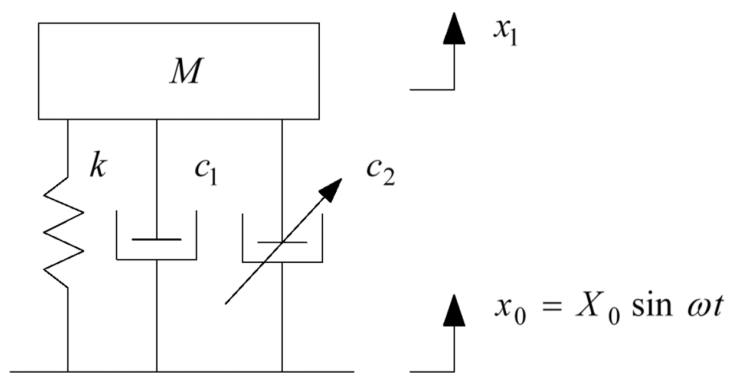

Fig. 1. Isolation system with mixed viscous damping under harmonic base excitation.

where $k$ is the spring constant, $c_{1}$ and $c_{2}$ are the damping constants, $x_{1}$ is the displacement response and $x_{0}$ is the base displacement. Given the relative displacement between mass $M$ and the base as $\delta=x_{1}-x_{0}$ and supposing a motion excitation as $x_{0}=X_{0} \sin \omega t$ where $\dot{x}_{0}=X_{0} \omega \cos \omega t$ and $\ddot{x}_{0}=-X_{0} \omega^{2} \sin \omega t$, we rewrite Eq. (1) as

$$
\ddot{\delta}+\frac{k}{M} \delta+\frac{c_{1}}{M} \dot{\delta}+\frac{c_{2}}{M}|\dot{\delta}| \dot{\delta}=X_{0} \omega^{2} \sin \omega t
$$

Introducing the following non-dimensional parameters

$$
\begin{aligned}
& \tau=\omega_{n} t, \quad \lambda=\frac{\omega}{\omega_{n}}, \quad \omega_{n}=\sqrt{\frac{k}{M}}, \quad \xi_{1}=\frac{c_{1}}{2 M \omega_{n}}, \quad \xi_{2}=\frac{c_{2} X_{0}}{M}, \quad y_{\delta}=\frac{\delta}{X_{0}}, \quad \dot{y}_{\delta}=\frac{\dot{\delta}}{X_{0} \omega_{n}}, \\
& \text { and } \ddot{y}_{\delta}=\frac{\ddot{\delta}}{X_{0} \omega_{n}^{2}},
\end{aligned}
$$

Equation (2) takes the form

$$
\ddot{y}_{\delta}+y_{\delta}+2 \xi_{1} \dot{y}_{\delta}+\xi_{2}\left|\dot{y}_{\delta}\right| \dot{y}_{\delta}=\lambda^{2} \sin \lambda \tau
$$

The first approximation to the steady-state solution of Eq. (3) based on the HBM $[13,16]$ is assumed in the form

$$
y_{\delta}(\tau)=Y_{0}+Y_{1} \sin (\lambda \tau-\varphi)=Y_{0}+Y_{1} \sin \phi
$$

where $\phi=\lambda \tau-\varphi$ and

$$
\begin{aligned}
& \dot{y}_{\delta}(\tau)=Y_{1} \lambda \cos \phi, \\
& \ddot{y}_{\delta}(\tau)=-Y_{1} \lambda^{2} \sin \phi
\end{aligned}
$$

The amplitude $Y_{1}$ is namely the relative transmissibility.

Let $f\left(y_{\delta}, \dot{y}_{\delta}\right)=y_{\delta}+2 \xi_{1} \dot{y}_{\delta}+\xi_{2}\left|\dot{y}_{\delta}\right| \dot{y}_{\delta}$, and substituting Eqs (5) and (6) into it yields

$$
f\left(y_{\delta}, \dot{y}_{\delta}\right)=Y_{0}+Y_{1} \sin \phi+2 \xi_{1} Y_{1} \lambda \cos \phi+\xi_{2} Y_{1}^{2} \lambda^{2}|\cos \phi| \cos \phi
$$

The function $f\left(y_{\delta}, \dot{y}_{\delta}\right)$ can be approximated by Fourier expansion up to the first order as

$$
f\left(y_{\delta}, \dot{y}_{\delta}\right) \approx a_{0}+a_{1} \cos \phi+a_{2} \sin \phi
$$

where the Fourier coefficients $a_{0}, a_{1}$ and $a_{2}$ are given by

$$
a_{0}=\frac{1}{2 \pi} \int_{0}^{2 \pi} f\left(y_{\delta}, \dot{y}_{\delta}\right) \mathrm{d} \phi=Y_{0}
$$




$$
\begin{aligned}
& a_{1}=\frac{1}{\pi} \int_{0}^{2 \pi} f\left(y_{\delta}, \dot{y}_{\delta}\right) \cos \phi \mathrm{d} \phi=2 \xi_{1} Y_{1} \lambda+\frac{8}{3 \pi} \xi_{2} Y_{1}^{2} \lambda^{2} \\
& a_{2}=\frac{1}{\pi} \int_{0}^{2 \pi} f\left(y_{\delta}, \dot{y}_{\delta}\right) \sin \phi \mathrm{d} \phi=Y_{1}
\end{aligned}
$$

Then, making use of Eqs (6) and (8) in Eq. (3) provides

$$
-Y_{1} \lambda^{2} \sin (\lambda \tau-\varphi)+Y_{0}+Y_{1} \sin (\lambda \tau-\varphi)+\left(2 \xi_{1} Y_{1} \lambda+\frac{8}{3 \pi} \xi_{2} Y_{1}^{2} \lambda^{2}\right) \cos (\lambda \tau-\varphi)=\lambda^{2} \sin \lambda \tau
$$

Equating the constant term and the coefficients of the same harmonics from both sides of Eq. (9), the steady-state condition can be obtained as

$$
\begin{aligned}
& Y_{0}=0 \\
& \left(Y_{1}-Y_{1} \lambda^{2}\right) \cos \varphi+\left(2 \xi_{1} Y_{1} \lambda+\frac{8}{3 \pi} \xi_{2} Y_{1}^{2} \lambda^{2}\right) \sin \varphi=\lambda^{2} \\
& -\left(Y_{1}-Y_{1} \lambda^{2}\right) \sin \varphi+\left(2 \xi_{1} Y_{1} \lambda+\frac{8}{3 \pi} \xi_{2} Y_{1}^{2} \lambda^{2}\right) \cos \varphi=0
\end{aligned}
$$

\subsection{ELDC and relative displacement transmissibility}

Combining Eqs (11) and (12), the following expression for frequency response is obtained which is an implicit function of the amplitude $Y_{1}$ (or the relative transmissibility) and the frequency ratio $\lambda$ :

$$
\left(1-\lambda^{2}\right)^{2} Y_{1}^{2}+\left(2 \xi_{1}+\frac{8}{3 \pi} \xi_{2} Y_{1} \lambda\right)^{2} \lambda^{2} Y_{1}^{2}=\lambda^{4}
$$

By introducing the ELDC

$$
\xi_{e}=\xi_{1}+\frac{4}{3 \pi} \xi_{2} Y_{1} \lambda
$$

the amplitude $Y_{1}$ can be obtained as

$$
Y_{1}=\frac{\lambda^{2}}{\sqrt{\left(1-\lambda^{2}\right)^{2}+4 \xi_{e}^{2} \lambda^{2}}}
$$

And according to Eq. (9), Eq. (3) can be rearranged as

$$
\ddot{y}_{\delta}+y_{\delta}+2 \xi_{e} \dot{y}_{\delta}=\lambda^{2} \sin \lambda \tau
$$

Substituting Eq. (15) into Eq. (14) gives

$$
\xi_{e}=\xi_{1}+\frac{4}{3 \pi} \xi_{2} \lambda^{3} \frac{1}{\sqrt{\left(1-\lambda^{2}\right)^{2}+4 \xi_{e}^{2} \lambda^{2}}}
$$

which is an implicit function of the ELDC $\xi_{e}$ and the frequency ratio $\lambda$.

Equation (16) has the form of the motion equation for a linear isolation system, and Eq. (15) gives the expression of $Y_{1}$ dependent on $\xi_{e}$ and $\lambda$, which is in agreement with the equation of the linear relative transmissibility. In Eq. (16), there is $2 \xi_{e}=2 \xi_{1}+\frac{8}{3 \pi} \xi_{2} Y_{1} \lambda$ where the first term $2 \xi_{1}$ and the second term $\frac{8}{3 \pi} \xi_{2} Y_{1} \lambda$ are corresponding to the linear and the quadratic damping terms, respectively. The expression $\frac{8}{3 \pi} \xi_{2} Y_{1} \lambda$ is consistent with the equivalent viscous damping coefficient $[4,14]$. So the conclusion is reached that the common used equivalent viscous damping coefficient in engineering has the first harmonic precision. 


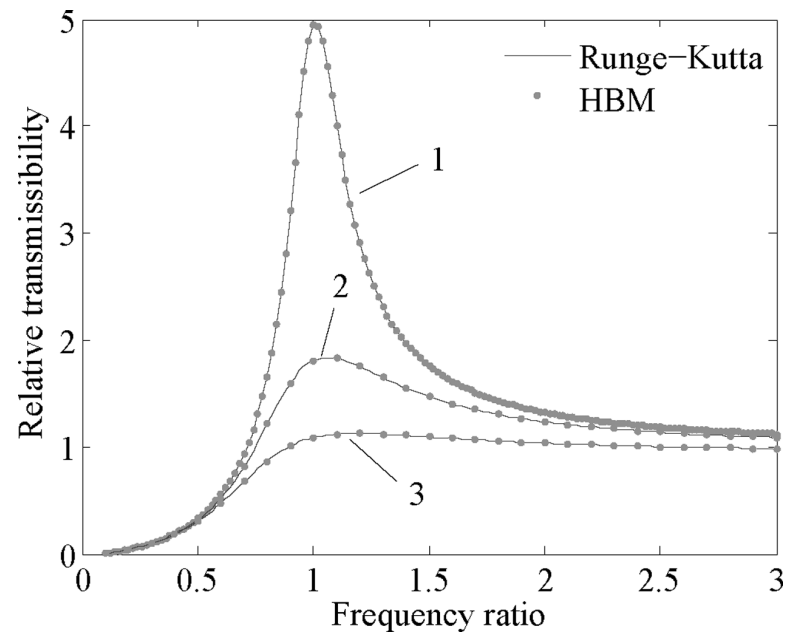

(a)

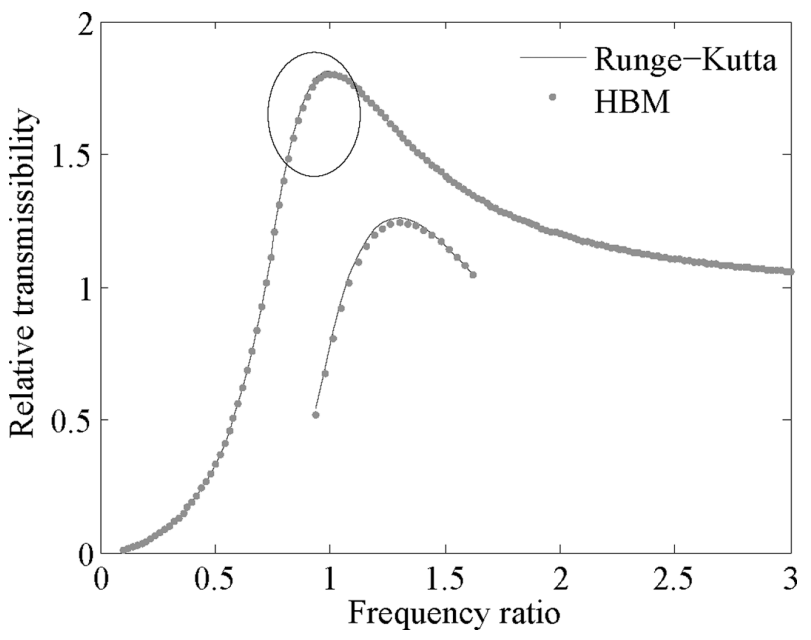

(b)

Fig. 2. Comparison of relative transmissibility by the HBM and the Runge-Kutta method. (a) $1-\xi_{1}=0.08 \xi_{2}=0.01 ; 2-\xi_{1}=0.2 \xi_{2}=0.1$; $3-\xi_{1}=0.3 \xi_{2}=0.35$; and (b) $\xi_{1}=0.01 \xi_{2}=0.35$.

\subsection{Absolute displacement transmissibility}

Besides relative displacement transmissibility, absolute displacement transmissibility is also the common index to evaluate the performance of isolation systems [14]. In this subsection, absolute displacement transmissibility is obtained as a function of the ELDC $\xi_{e}$ and the frequency ratio $\lambda$.

According to Subsection 2.1, the first order harmonic solution of Eq. (3) is

$$
y_{\delta}(\tau)=Y_{1} \sin (\lambda \tau-\varphi)
$$

where $Y_{1}$ is determined by Eq. (15).

Using Eqs (12) and (14), the expression of phase angle $\varphi$ is obtained as

$$
\varphi=\arctan \left(\frac{2 \xi_{e} \lambda}{1-\lambda^{2}}\right)
$$

Besides, $y_{\delta}$ can be written as

$$
y_{\delta}=z(\tau)-\sin \lambda \tau
$$

where $z(\tau)=\frac{x_{1}}{X_{0}}$

Assuming $z(\tau)=Z_{1} \sin (\lambda \tau-\theta)$ in the steady state, the amplitude $Z_{1}$ is actually the absolute transmissibility. According to Eq. (17), the ELDC $\xi_{e}$ is independent on amplitudes of the excitation and the response, so by the linear vibration theory the absolute transmissibility $Z_{1}$ is

$$
Z_{1}=\sqrt{\frac{1+\left(2 \xi_{e} \lambda\right)^{2}}{\left(1-\lambda^{2}\right)^{2}+\left(2 \xi_{e} \lambda\right)^{2}}}
$$

\section{Comparisons of the HBM solutions and the numerical solutions for displacement transmissibility (frequency responses)}

The HBM solutions and the numerical solutions of the fourth order Runge-Kutta method are compared in Fig. 2 for relative transmissibility and in Fig. 3 for absolute transmissibility, respectively. The HBM results are obtained using Eqs (14), (15) and (21). And the transmissibility curves are gotten by the Runge-Kutta method in terms of the maximum amplitudes of the non-dimensional displacement $y_{\delta}$ according to Eq. (3) and $z(\tau)$ according to Eq. (20) 


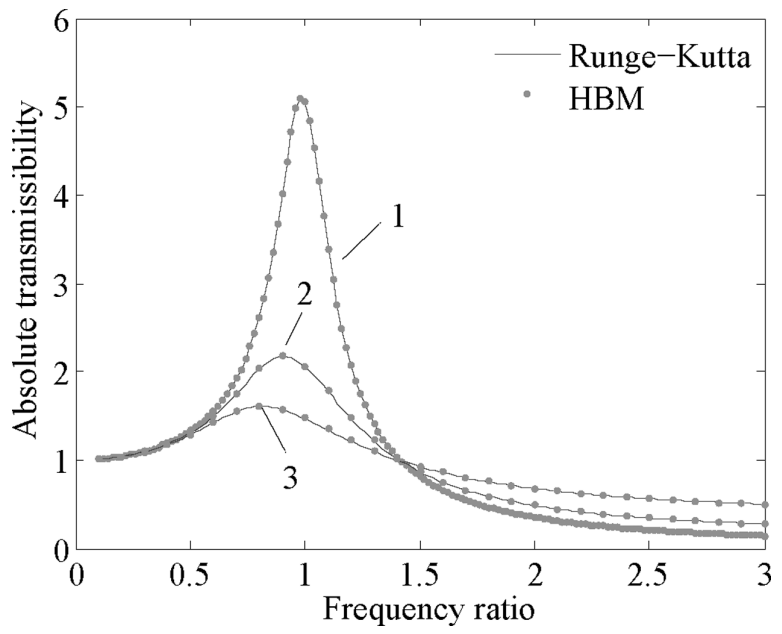

(a)

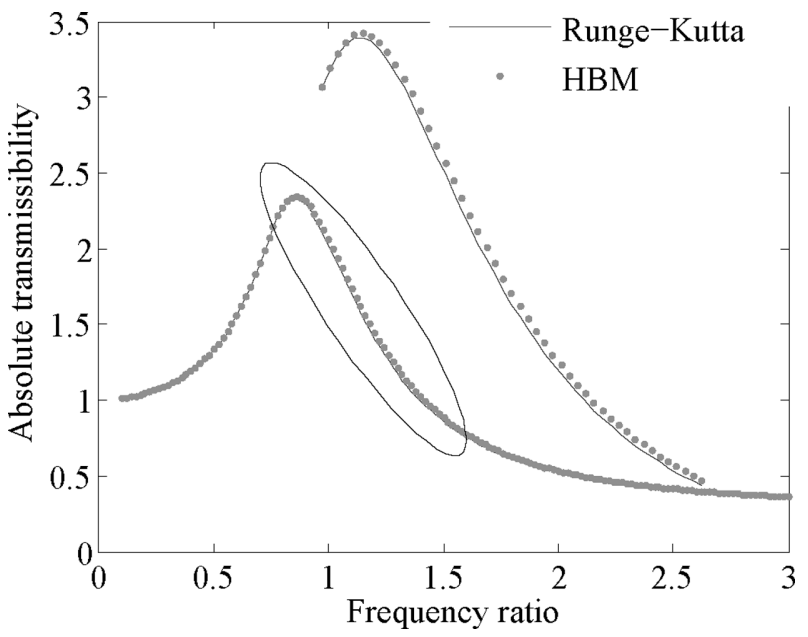

(b)

Fig. 3. Comparison of absolute transmissibility by the HBM and the Runge-Kutta method. (a) $1-\xi_{1}=0.08 \xi_{2}=0.01 ; 2-\xi_{1}=0.2 \xi_{2}=0.1$; $3-\xi_{1}=0.3 \xi_{2}=0.35$; and (b) $\xi_{1}=0.01 \xi_{2}=0.35$.

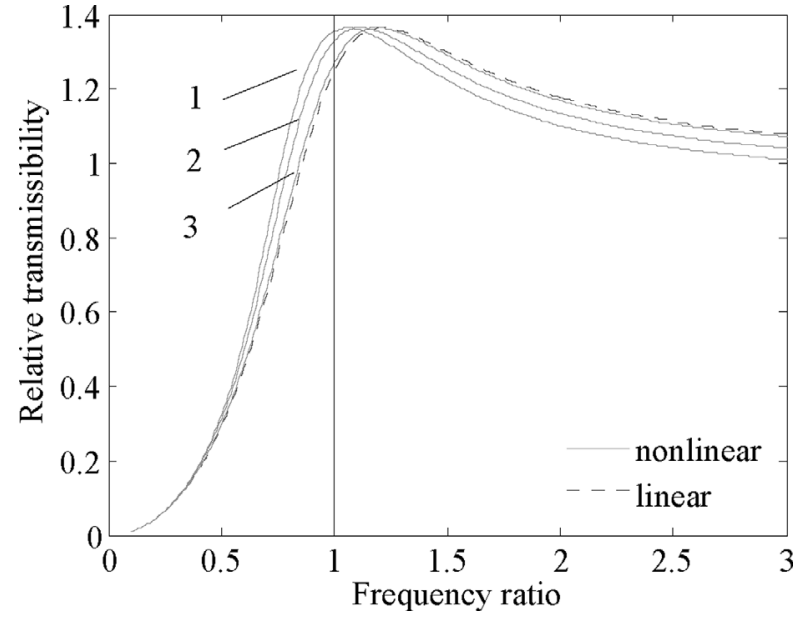

Fig. 4. Relative transmissibility with the corresponding damping ratio of linear system equal to $0.4 .1-\xi_{1}=0.139 \xi_{2}=0.4 ; 2-\xi_{1}=0.25$ $\xi_{2}=0.223 ; 3-\xi_{1}=0.37 \xi_{2}=0.043$.

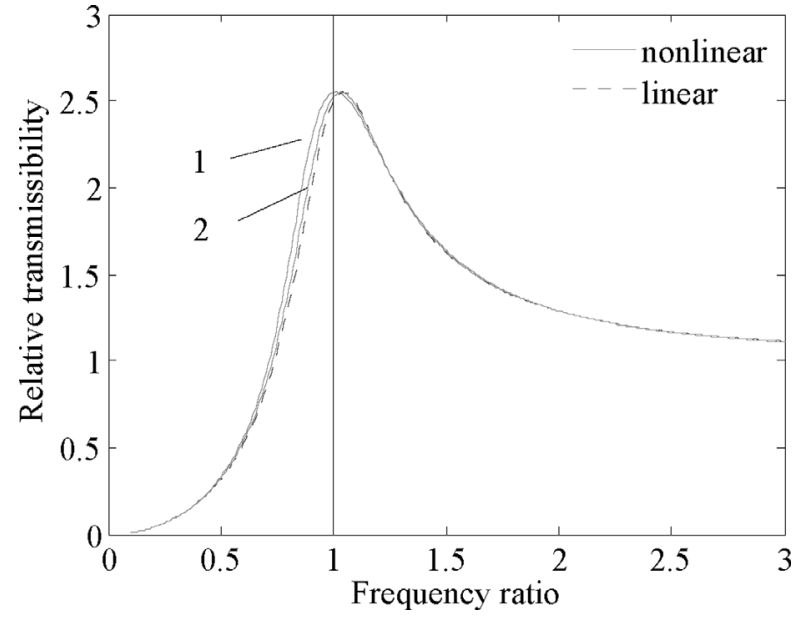

Fig. 5. Relative transmissibility with the corresponding damping ratio of linear system equal to 0.2. $1-\xi_{1}=0.05 \xi_{2}=0.135$; $2-\xi_{1}=0.153 \xi_{2}=0.0418$.

in the steady state for each certain frequency ratio $\lambda$. The range of frequency ratios is from 0.1 to 3 with interval 0.02 and the duration of simulation is $\tau=300$. It is observed that analytical solutions have good agreement with numerical results for both relative and absolute transmissibility curves in weakly and strongly nonlinear cases, and when the nonlinear damping component is obviously predominant in the mixed damping force, there are small offsets near the resonant frequency respectively shown in Figs 2(b) and 3(b).

The comparisons show that the analytical solutions of displacement transmissibility are able to represent response characteristics of the system with fairly good accuracy.

\section{Influence of the nonlinear damping component}

Based on the analytical solutions given in Eqs (15) and (21), frequency responses for different values of parameters $\xi_{1}$ and $\xi_{2}$ are discussed in contrast with the linear damping system having the same peak transmissibility to study the effects of the nonlinear damping term. 


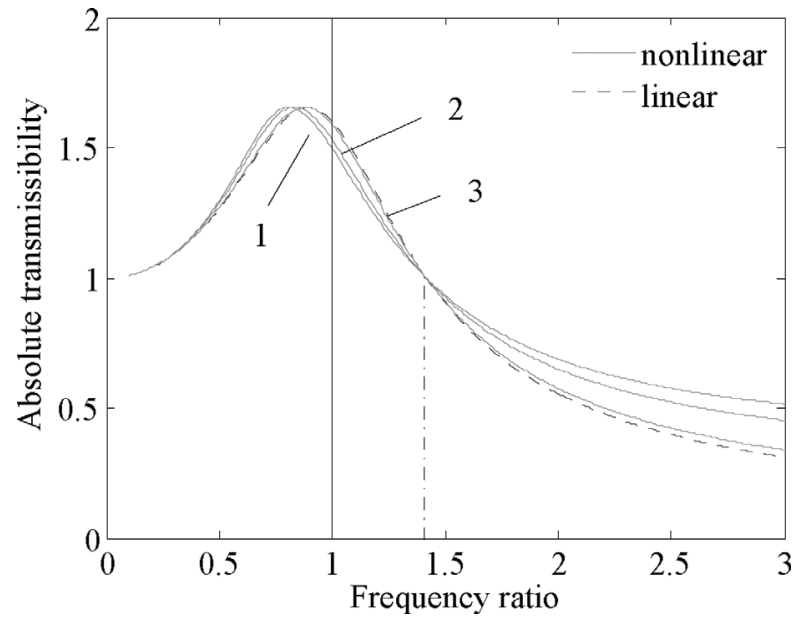

Fig. 6. Absolute transmissibility with the corresponding damping ratio of linear system equal to $0.4 .1-\xi_{1}=0.25 \xi_{2}=0.425 ; 2-\xi_{1}=0.3$ $\xi_{2}=0.272 ; 3-\xi_{1}=0.38 \xi_{2}=0.05$.

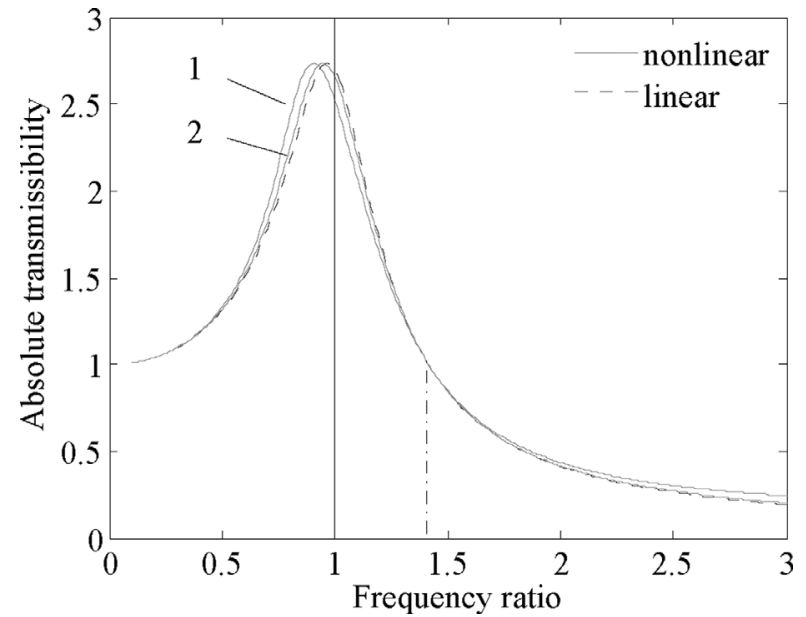

Fig. 7. Absolute transmissibility with the corresponding damping ratio of linear system equal to $0.2 .1-\xi_{1}=0.05 \xi_{2}=0.17$; $2-\xi_{1}=0.162 \xi_{2}=0.04$.

\subsection{On relative displacement transmissibility}

Figures 4 and 5 depict the frequency responses of the relative displacement for nonlinear damper isolation systems with different damping magnitudes compared with a linear isolator in two cases. The damping parameters $\xi_{1}$ and $\xi_{2}$ are chosen in such a way that the nonlinear and linear damping systems provide the same peak relative transmissibility of the isolated mass $M$. It can be seen that the peak values of the curves for both weakly and strongly nonlinear damping occur at lower frequencies compared with the case of the linear damping and that the stronger the nonlinear damping component is in the damping force $f_{d}\left(\dot{y}_{\delta}\right)=2 \xi_{1} \dot{y}_{\delta}+\xi_{2}\left|\dot{y}_{\delta}\right| \dot{y}_{\delta}$, the larger the shift is. It is also noted that the nonlinear damping model produces lower relative transmissibility at frequencies above the resonance frequency of the corresponding linear model as the damping ratio of the linear model is bigger (Fig. 4), while it is not obvious in Fig. 5 with a smaller damping ratio of the linear model.

\subsection{On absolute displacement transmissibility}

The analysis of the absolute transmissibility is in a similar way to that of the relative transmissibility. Comparing nonlinear damping models with different values of parameters $\xi_{1}$ and $\xi_{2}$ with corresponding linear models in Figs 6 and 7 , it is observed that, as the same with the case of relative transmissibility, the resonance frequency also shifts left due to the effect of the nonlinear damping in contrast with the linear isolator and the curve involving a stronger nonlinear damping also has a more visible deflection. Meanwhile, the nonlinear damping model produces increased transmissibility for the frequency ratio $\lambda>\sqrt{2}$, which, however, is not so distinct as the damping ratio of the corresponding linear system is in a relatively low magnitude (Fig. 7).

The trends illustrated in this subsection are consistent with the results obtained by Popov and Sankar [8] for a nonlinear orifice type damper with the SDOF model as Fig. 1 using the Runge-Kutta method. The quadratic damping coefficient was inversely proportional to the non-dimensional diameter of the orifice. The nonlinear damping caused a significant shift in resonant frequency and obviously increased transmissibility for $\lambda>\sqrt{2}$ compared with the linear damping case when the non-dimensional diameter of the orifice was smaller.

\section{Isolation system with a type of piecewise mixed viscous damping}

\subsection{Model and approximate solution}

We consider piecewise mixed viscous damping as 


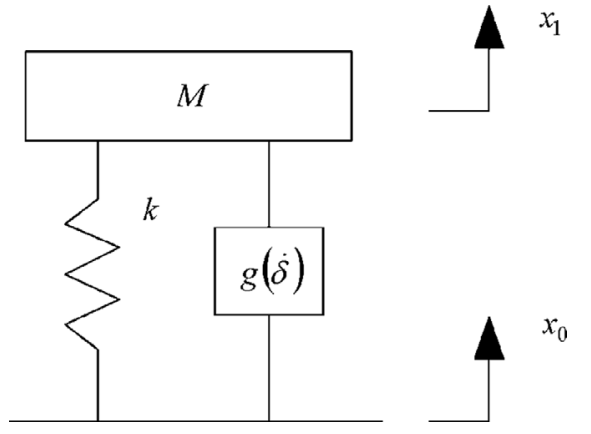

Fig. 8. Base isolation system with piecewise damping force.

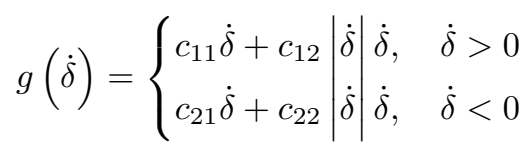

If the damper or isolator presents this type of damping characteristic according to the sign of the relative velocity, we can also study its response characteristics in the same way as the above analysis of the isolation system in Fig. 1. The governing differential equation of the system with the piecewise damping shown in Fig. 8 after nondimensionalization is

$$
\ddot{y}_{\delta}+y_{\delta}+G\left(\dot{y}_{\delta}\right)=\lambda^{2} \sin \lambda \tau
$$

where

$$
G\left(\dot{y}_{\delta}\right)= \begin{cases}2 \xi_{11} \dot{y}_{\delta}+\xi_{12}\left|\dot{y}_{\delta}\right| \dot{y}_{\delta}, & \dot{y}_{\delta} \geqslant 0 \\ 2 \xi_{21} \dot{y}_{\delta}+\xi_{22}\left|\dot{y}_{\delta}\right| \dot{y}_{\delta}, & \dot{y}_{\delta}<0\end{cases}
$$

and $\xi_{11}=\frac{c_{11}}{2 M \omega_{n}}, \xi_{21}=\frac{c_{21}}{2 M \omega_{n}}, \xi_{12}=\frac{c_{12} X_{0}}{M}, \xi_{22}=\frac{c_{22} X_{0}}{M}$.

Sending

$$
f\left(y_{\delta}, \dot{y}_{\delta}\right)=y_{\delta}+G\left(\dot{y}_{\delta}\right)
$$

and assuming a steady-state solution for Eq. (23) in the form of Eq. (4) as well, Eq. (25) becomes

$$
f\left(y_{\delta}, \dot{y}_{\delta}\right)=y_{\delta}+ \begin{cases}2 \xi_{11} \dot{y}_{\delta}+\xi_{12}\left|\dot{y}_{\delta}\right| \dot{y}_{\delta}, & -\frac{\pi}{2} \leqslant \phi \leqslant \frac{\pi}{2} \\ 2 \xi_{21} \dot{y}_{\delta}+\xi_{22}\left|\dot{y}_{\delta}\right| \dot{y}_{\delta}, & \frac{\pi}{2}<\phi<\frac{3 \pi}{2}\end{cases}
$$

By Fourier expansion as Eq. (8), the Fourier coefficients $a_{0}, a_{1}$ and $a_{2}$ are obtained:

$$
\begin{aligned}
& a_{0}=\frac{1}{2 \pi} \int_{-\frac{\pi}{2}}^{\frac{3 \pi}{2}} f\left(y_{\delta}, \dot{y}_{\delta}\right) \mathrm{d} \phi=Y_{0}+\frac{2}{\pi}\left(\xi_{11}-\xi_{21}\right) Y_{1} \lambda+\frac{1}{4}\left(\xi_{12}-\xi_{22}\right) Y_{1}^{2} \lambda^{2} \\
& a_{1}=\frac{1}{\pi} \int_{-\frac{\pi}{2}}^{\frac{3 \pi}{2}} f\left(y_{\delta}, \dot{y}_{\delta}\right) \cos \phi \mathrm{d} \phi=\left(\xi_{11}+\xi_{21}\right) Y_{1} \lambda+\frac{4}{3 \pi}\left(\xi_{12}+\xi_{22}\right) Y_{1}^{2} \lambda^{2} \\
& a_{2}=\frac{1}{\pi} \int_{-\frac{\pi}{2}}^{\frac{3 \pi}{2}} f\left(y_{\delta}, \dot{y}_{\delta}\right) \sin \phi \mathrm{d} \phi=Y_{1}
\end{aligned}
$$


Then, Eq. (23) can be rewritten as

$$
\begin{aligned}
& -Y_{1} \lambda^{2} \sin \phi+\left(Y_{0}+\frac{2}{\pi}\left(\xi_{11}-\xi_{21}\right) Y_{1} \lambda+\frac{1}{4}\left(\xi_{12}-\xi_{22}\right) Y_{1}^{2} \lambda^{2}\right) \\
& +\left(\left(\xi_{11}+\xi_{21}\right) Y_{1} \lambda+\frac{4}{3 \pi}\left(\xi_{12}+\xi_{22}\right) Y_{1}^{2} \lambda^{2}\right) \cos \phi+Y_{1} \sin \phi=\lambda^{2} \sin (\phi+\varphi)
\end{aligned}
$$

which leads to

$$
\begin{aligned}
& Y_{0}=-\left(\frac{2}{\pi}\left(\xi_{11}-\xi_{21}\right)+\frac{1}{4}\left(\xi_{12}-\xi_{22}\right) Y_{1} \lambda\right) Y_{1} \lambda \\
& Y_{1}\left(1-\lambda^{2}\right)=\lambda^{2} \cos \varphi \\
& \left(\xi_{11}+\xi_{21}\right) Y_{1} \lambda+\frac{4}{3 \pi}\left(\xi_{12}+\xi_{22}\right) Y_{1}^{2} \lambda^{2}=\lambda^{2} \sin \varphi
\end{aligned}
$$

Using Eqs (29) and (30) results in

$$
\begin{aligned}
& \left(1-\lambda^{2}\right)^{2} Y_{1}^{2}+\left(\left(\xi_{11}+\xi_{21}\right)+\frac{4}{3 \pi}\left(\xi_{12}+\xi_{22}\right) Y_{1} \lambda\right)^{2} Y_{1}^{2} \lambda^{2}=\lambda^{4} \\
& \varphi=\arctan \left(\frac{\left(\xi_{11}+\xi_{21}\right) \lambda+\frac{4}{3 \pi}\left(\xi_{12}+\xi_{22}\right) Y_{1} \lambda^{2}}{1-\lambda^{2}}\right)
\end{aligned}
$$

Introducing the ELDC as

$$
\xi_{e}=\frac{1}{2}\left(\xi_{11}+\xi_{21}\right)+\frac{2}{3 \pi}\left(\xi_{12}+\xi_{22}\right) Y_{1} \lambda
$$

and substituting it into Eqs (31) and (32), the response amplitude (relative displacement transmissibility) $Y_{1}$ and the phase angle $\varphi$ are given in the same forms of Eqs (15) and (19), respectively. Taking into account Eq. (28), $Y_{0}=0$ occurs only when $\xi_{11}=\xi_{21}$ and $\xi_{12}=\xi_{22}$, which is in the non-piecewise case in Section 2. Therefore, the first steady-state approximation of the Eq. (23) is

$$
y_{\delta}(\tau)=Y_{0}+Y_{1} \sin (\lambda \tau-\varphi)
$$

where $Y_{0}$ is not zero, which indicates that the piecewise damping given in Eq. (22) leads to the change of equilibrium position of the isolation system.

For the absolute displacement transmissibility $Z_{1}$, here still suppose it in the form of Eq. (21). In the next subsection, we prove the accuracy of the supposition by comparing it with the numerical solution.

\subsection{Numerical simulation for absolute displacement transmissibility}

The absolute transmissibility curves of four different cases obtained by the fourth order Runge-Kutta method and the analytical method are compared in Fig. 9. The results of every case show good agreement, which verifies the supposition in the Subsection 5.1.

Based on Eq. (33), displacement transmissibility is characterized by the parameters $\xi_{11}+\xi_{21}$ and $\xi_{12}+\xi_{22}$. The system described by Eq. (23) is not prone to an instability named chatter because of the relative velocity whereby the damping force is zero at the instant the damper is switched [12]. Therefore, the displacement response characteristics can be analysed as similar to the case of Eq. (3). 


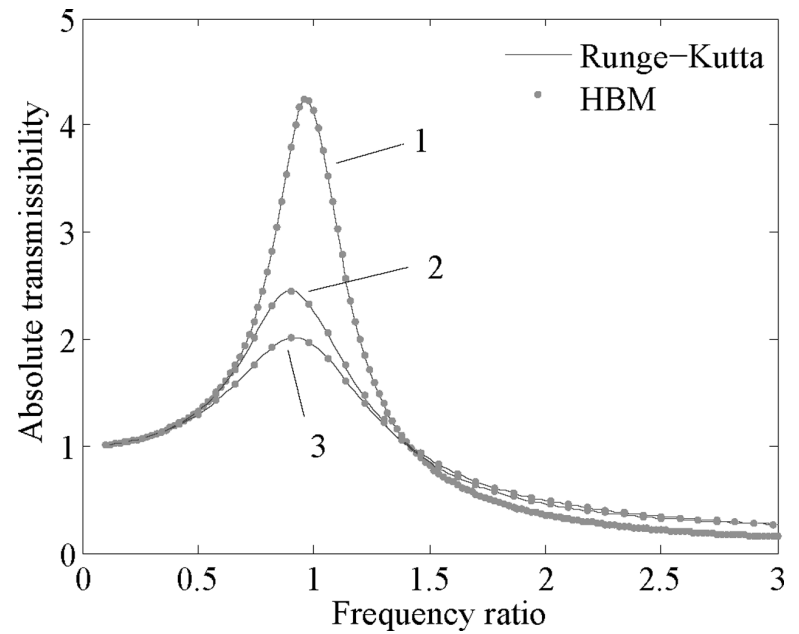

(a)

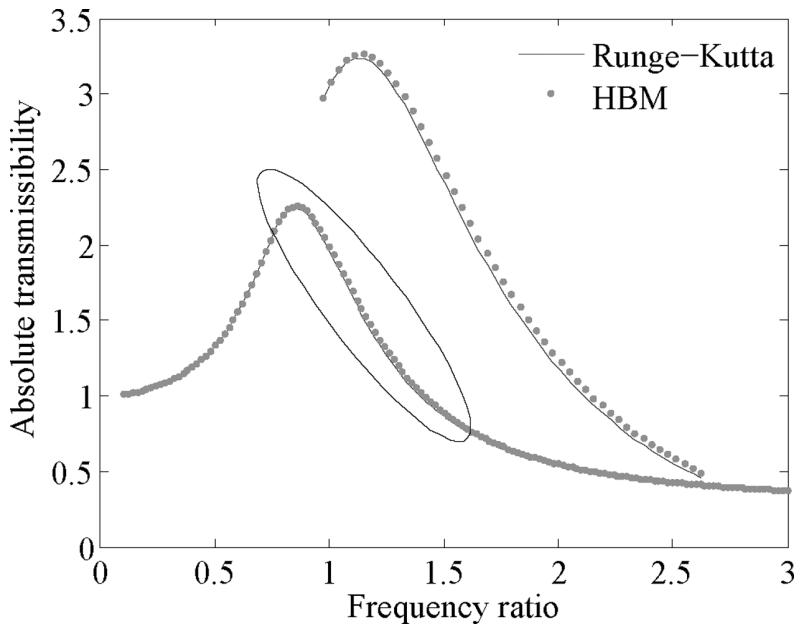

(b)

Fig. 9. Comparison of absolute transmissibility by the numerical and analytical methods. (a) $1-\xi_{11}=0.05 \xi_{12}=0.02 \xi_{21}=0.08$ $\xi_{22}=0.05 ; 2-\xi_{11}=0.02 \xi_{12}=0.3 \xi_{21}=0.2 \xi_{22}=0.01 ; 3-\xi_{11}=0.25 \xi_{12}=0.02 \xi_{21}=0.3 \xi_{22}=0.05$; (b) $\xi_{11}=0.02$ $\xi_{12}=0.4 \xi_{21}=0.05 \xi_{22}=0.3$.

\section{Conclusions}

The base isolation system with nonlinear damping force as $f_{d}(\dot{x})=c_{1} \dot{x}+c_{2}|\dot{x}| \dot{x}$ which sometimes can represent the damping characteristic of dampers and isolators in practical engineering is studied using the HBM and the first approximate solutions are deduced. By introducing the ELDC, the motion equation and the response characteristics including the relative and absolute displacement transmissibility and the phase angle can all be expressed as functions of the ELDC in the same forms of a linear isolation system. The component of the ELDC corresponding to the quadratic damping term is consistent with the equivalent viscous damping coefficient based on equivalent energy dissipation. In accordance with the numerical solutions, the analytical results have high accuracy. And further, the performance characteristics of the nonlinear damping isolators are evaluated analytically from the viewpoint of the displacement transmissibility for different values of damping coefficients with equal peak transmissibility against those of the linear system. Finally, an ideal isolator with piecewise mixed viscous damping by the sign of the relative velocity is investigated employing the same approach as above, which is found that the displacement response characteristics can also be studied by introducing the ELDC fairly well.

\section{Acknowledgments}

The authors acknowledge the Science and Technology Support Plan of Jiangsu China (Approval No. BE2010047).

\section{References}

[1] P.F. Guo, Z.Q. Lang and Z.K. Peng, Analysis and design of the force and displacement transmissibility of nonlinear viscous damper based vibration isolation systems, Nonlinear Dynamics 67 (2012), 2671-2687.

[2] Y. Starosvetsky and O.V. Gendelman, Vibration absorption in systems with a nonlinear energy sink: Nonlinear damping, Journal of Sound and Vibration 324 (2009), 916-939.

[3] R.D. Eyres, A.R. Champneys and N.A.J. Lieven, Modelling and dynamic response of a damper with relief valve, Nonlinear Dynamics 40 (2005), 119-147.

[4] A. Rittweger, J. Albus, E. Hornung, H. Ory and P. Mourey, Passive damping devices for aerospace structures, Acta Astronautica 50 (2002), 597-608.

[5] A. Geisberger, A. Khajepour and F. Golnaraghi, Non-linear modeling of hydraulic mounts: Theory and experiment, Journal of Sound and Vibration 249 (2002), 371-397. 
[6] J. Lee and K. Kim, An efficient technique for design of hydraulic engine mount via design variable-embedded damping modeling, ASME Journal of Vibration and Acoustics 127 (2005), 93-99.

[7] M. Kuzukawa and T. Tanaka, Vibration dampening device with an elastic body and viscous liquid, U.S. Patent No. 5,707,048, 1998.

[8] G. Popov and S. Sankar, Modeling and analysis of non-linear orifice type damping in vibration isolators, Journal of Sound and Vibration 183 (1995), 751-764.

[9] J. Wallaschek, Dynamics of non-linear automobile shock-absorbers, International Journal of Non-Linear Mechanics 25(2/3) (1990), 299_ 308.

[10] X. Sun and J. Zhang, Performance of earth-moving machinery cab with hydraulic mounts in low frequency, Journal of Vibration and Control published online (28 Nov 2012), DOI: 10.1177/1077546312464260.

[11] A.K. Mallik, V. Kher, M. Puri and H. Hatwal, On the modeling of non-linear elastomeric vibration isolators, Journal of Sound and Vibration 219(2) (1999), 239-253.

[12] T.P. Waters, Y. Hyun and M.J. Brennan, The effect of dual-rate suspension damping on vehicle response to transient road inputs, ASME Journal of Vibration and Acoustics 131(1) (2009), Article Number: 011004.

[13] H. Nayfeh and D.T. Mook, Nonlinear Oscillations, John Wiley \& Sons, New York, 1979.

[14] C.M. Harris, Shock and Vibration Handbook, McGraw-Hill, New York, 1987.

[15] A.K. Mallik, Forced harmonic vibration of a Duffing oscillator with different damping mechanisms, in: The Duffing Equation: Nonlinear Oscillators and Their Behavior, I. Kovacic and M. J. Brennan, eds, John Wiley and Sons, Chichester, West Sussex, (2011), 175-217.

[16] B. Ravindra and A.K. Mallik, Performance of non-linear vibration isolators under harmonic excitation, Journal of Sound and Vibration 170 (1994), 325-337.

[17] N. Eslaminasab, O. Vahid and F. Golnaraghi, Nonlinear analysis of switched semi-active controlled systems, Vehicle System Dynamics 49(1-2) (2011), 291-309.

[18] R. Alkhatib, G.N. Jazar and M.F. Golnaraghi, Optimal design of passive linear suspension using genetic algorithm, Journal of Sound and Vibration 275 (2004), 665-691.

[19] Z. Milovanovic, I. Kovacic and M.J. Brennan, On the displacement transmissibility of a base excited viscously damped nonlinear vibration isolator, ASME Journal of Vibration and Acoustics 131(5) (2009), Article Number: 054502. 

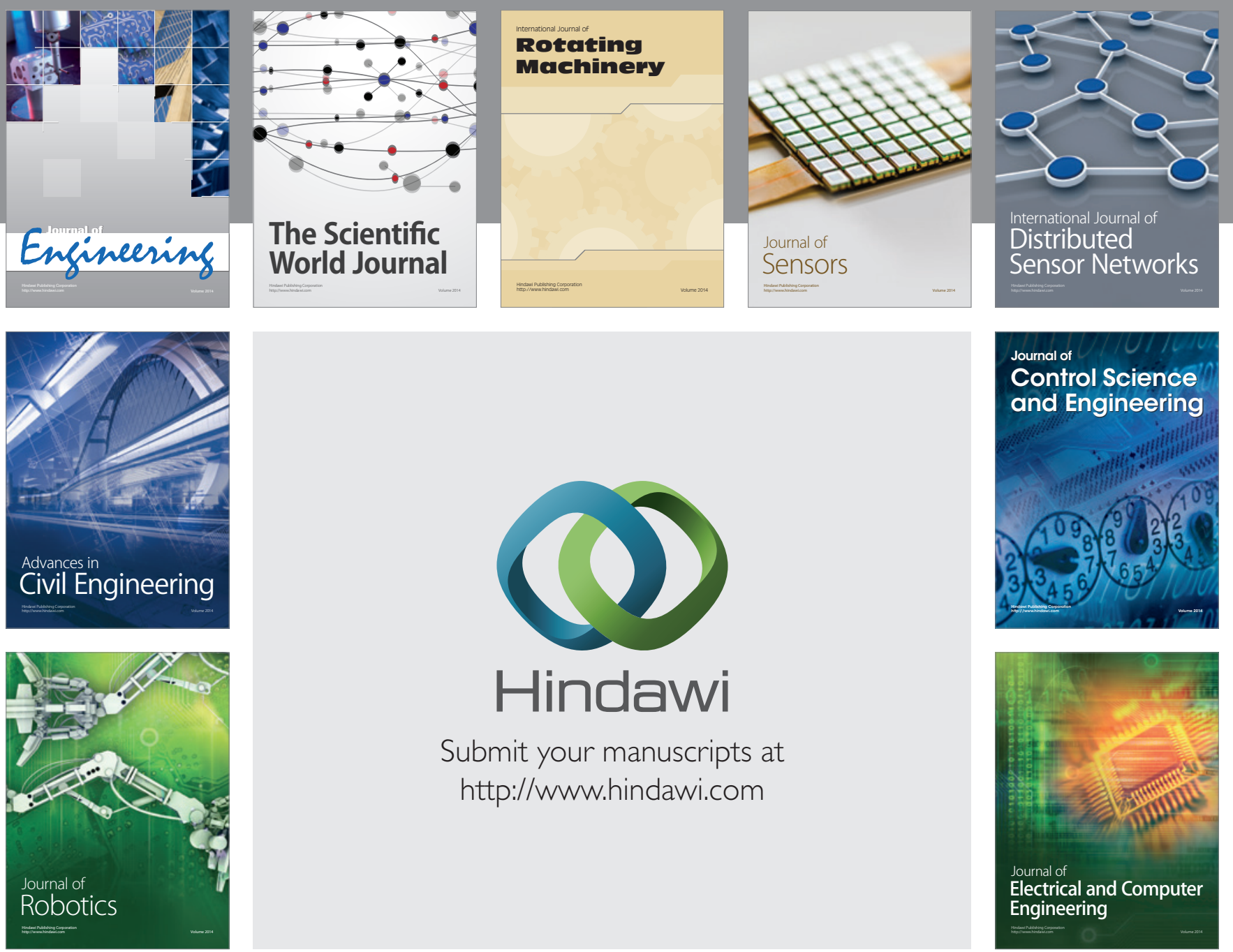

Submit your manuscripts at

http://www.hindawi.com
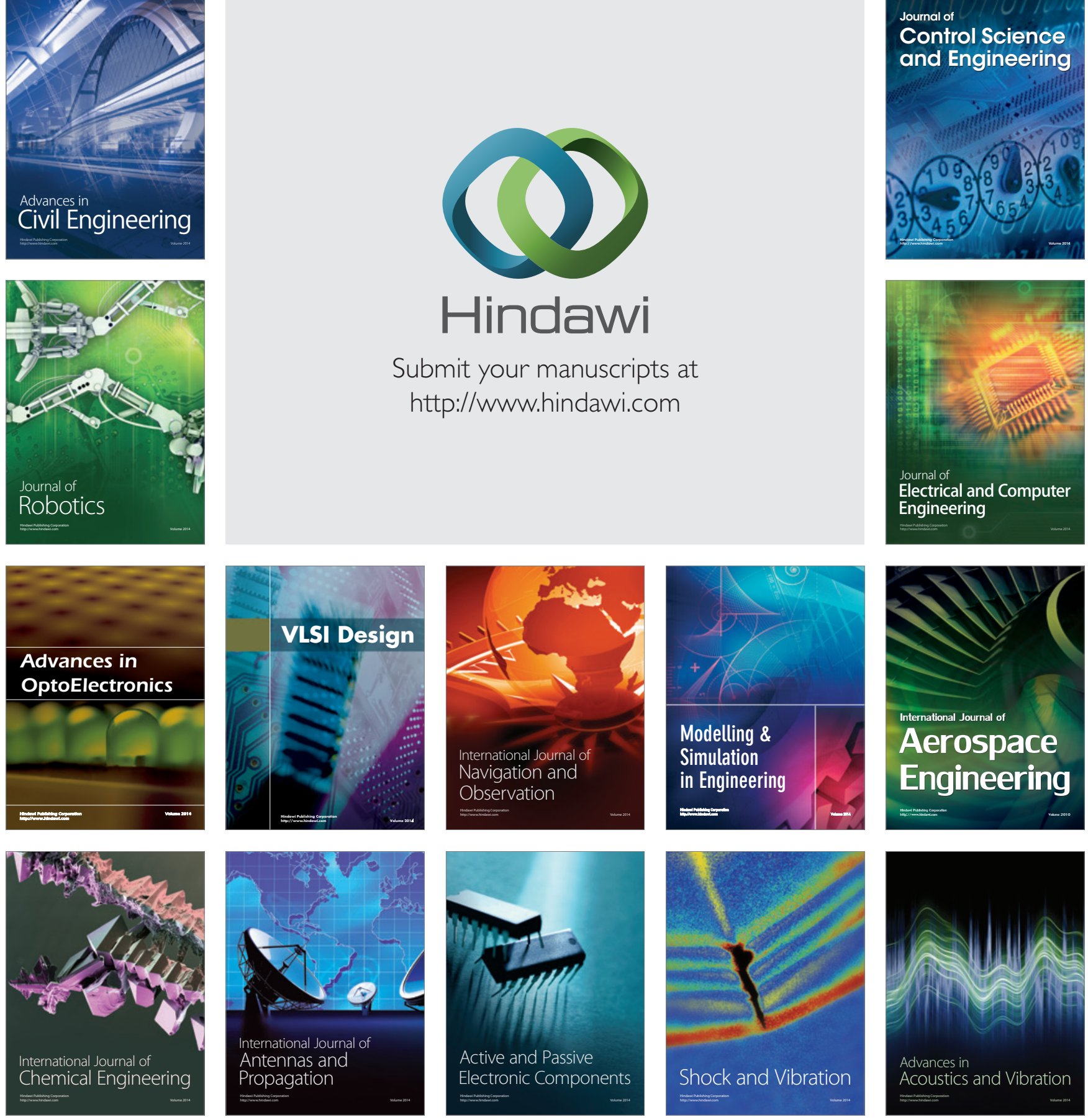